\title{
Adjuvant therapy for pancreatic cancer: current status and future directions
}

\author{
J. L. Van Laethem' ', K. Haustermans², P. Hammel ${ }^{3}$, J. Closset' ${ }^{1}$, J. F. Gigot ${ }^{4}$, C. Penna ${ }^{5}$, B. Nordlinger 5 , \\ P. Van Houtte ${ }^{6}$, E. Van Cutsem ${ }^{2}$, V. Budach ${ }^{7}$
}

Pancreatic cancer is one of the most lethal cancers and has a global survival rate of less than $5 \%$ at 5 years. Surgical resection remains the only curative measure but provides a median survival of only 12-15 months, due to the very high rates of both local recurrence and distant metastases ${ }^{1-3}$.

On the basis of this natural history, multiple adjuvant and more recently neoadjuvant therapies have been investigated in order to improve survival. This review is a critical assessment of the results and limitations of such trials and the authors discuss a rationale for the introduction of new developments in the (neo)adjuvant setting of pancreatic cancer.

\section{Postoperative adjuvant therapy}

The number of large randomised trials remains relatively limited The trials respectively conducted by the Gastrointestinal Tract cancer Study Group (GITSG) and the EORTC (40891) evaluated the addition of external-beam radiation therapy (EBRT) and concomitant 5FU as radiosensitizer after surgical resection (Table I) ${ }^{4}, 5$. In both trials, radiation consisted of 40 Gy given as a split course and 5FU given during weeks 1 and 5 The GITSG trial demonstrated a survival advantage for this multimodal therapy compared to resection alone (20 vs 11 months) but only included 43 patients $^{4}$. Ever since, adjuvant

\footnotetext{
1 Medico-surgical Department of Gastroenterology. Digestive Oncology Unit. Erasme University Hospital. Brussels (Belgium)

2 Departments of Radiotherapy and Internal Medicine. UZ Gasthuisberg. Leuven (Belgium)

3 Department of Gastroenterology. Hôpital Beaujon. Clichy (France)

4 Department of Hepatobiliary surgery. Cliniques Universitaires St Luc. Brussels (Belgium)

5 Department of Digestive and Oncologic Surgery. Hopital Ambroise Paré. Boulogne (France)

6 Department of Radiotherapy. Institut Jules Bordet. Brussels (Belgium)

7 Department of Radiotherapy. Charité University Clinics. Berlin (Germany)
}

chemoradiation with $5 \mathrm{FU}$ as radiosensitizer has been considered in the US as standard of care after curative surgery. Other US data derived from non-randomized studies also suggest that adjuvant chemoradiation may prolong median survival (19.5 vs 13.5 months for historic surgical controls) and this treatment has been identified as a favourable prognostic factor $3,6,7$. Moreover it has been suggested that continuous infusionnal 5FU should be preferably used in this setting as there was no significant toxicity observed 7 .

Between 1987 and 1995, 218 patients were randomized in the EORTC trial to receive either surgery followed by chemoradiation (40 Gy in a split course + a 5FU infusion during $2 \times 5$ days) or no further treatment after surgery ${ }^{5}$. Of these, 114 patients had pancreatic head cancer and the rest have periampullary tumors. The subgroup of pancreatic cancer patients who had multimodal treatment displayed a trend towards longer median survival than those who had surgery alone (17 vs 12.6 months) but the study was not sufficiently powered to demonstrate a statistically significant benefit Consequently, this adjuvant regimen was not considered as a standard treatment and, in Europe, surgery alone remains the standard care $^{5}$.

In a Norwegian study, the role of AMF (Adriamycin, Mitomycin and 5FU) chemotherapy was investigated in the adjuvant setting; this study enrolled 61 patients and showed a significant prolongation of median survival but no difference at 5 years $^{8}$.

All these trials raised challenging questions regarding either the methodology or the treatment regimen used: a) 20 to $24 \%$ of the assessable patients did not receive the intended treatment because of patient refusal, prolonged recovery after Whipple's resection or rapid tumour progression, thus introducing a bias into the results; b) no stratification was made between pancreatic and periampullary tumors which were mixed up at enrolment; c) the retroperitoneal margins were not accurately determined; d) compared to continuous radiation and 5FU administration, the treatment regimens, comprising split course radiation and $5 \mathrm{FU}$ bolus, cannot be considered optimal; e) there was no quality control/standardization of surgery and radiotherapy.

Details were recently published of the ESPAC-1 trial, the largest trial so far conducted in the setting of adjuvant therapy for pancreatic cancer'; this trial assessed the relative ro- 
TABLE I

Adjuvant chemoradiation studies in patients with resectable pancreatic cancer

\begin{tabular}{|c|c|c|c|c|}
\hline Author (year) (reference) & No of patients & Randomization & Treatment & Median survival (months) \\
\hline GITSG (1985) & 21 & \multirow[t]{2}{*}{ Yes } & $\mathrm{S}+40$ Gy split + 5FU b & $20^{*}$ \\
\hline (4) & 22 & & $\mathrm{~S}$ & 11 \\
\hline EORTC (1999) & 60 & \multirow[t]{3}{*}{ Yes } & $\mathrm{S}+40$ Gy split + 5FU 2x5 & 17.1 \\
\hline (5) & 54 & & days & 12.6 \\
\hline ESPAC-1 (2001) & 69 & & $\mathrm{~S}$ & \multirow{2}{*}{17.8} \\
\hline \multirow[t]{7}{*}{ (9) } & 74 & $2 \times 2$ & $\mathrm{~S}+5 \mathrm{FU} / \mathrm{FA}$ & \\
\hline & 70 & \multirow[t]{2}{*}{ factorial } & $\mathrm{S}+40$ Gy split + 5FU b & \multirow{2}{*}{15.8} \\
\hline & 72 & & $\mathrm{~S}+$ both & \\
\hline & 175 & pooled & chemoradiotherapy & 15.5 \\
\hline & 178 & randomization & no chemoradiatherapy & 16.1 \\
\hline & 238 & pooled & chemotherapy & $19.7^{*}$ \\
\hline & 235 & randomization & no chemotherapy & 14 \\
\hline Yeo (1997) & 120 & no & $\mathrm{S}+40-55 \mathrm{~Gy}+5 \mathrm{FU}$ & $19.5^{*}$ \\
\hline (3) & 53 & & S & 13.5 \\
\hline
\end{tabular}

*p significant; S: surgery; $5 \mathrm{FU}$ b: bolus 500 mg, 5FU c: continuous infusion $200-250 \mathrm{mg} / \mathrm{m}^{2} /$ day (d)

le of chemotherapy (bolus $5 \mathrm{FU}+$ folinic acid, 5 days monthly), chemoradiation (2x20 Gy over 2 weeks with bolus $5 \mathrm{FU})$ or chemoradiation followed by chemotherapy versus surgery alone in 541 patients $^{9}$ (Table I). This trial, however, was submitted to multiple criticisms for its study design, interpretation of data and control of the quality of the ressources used; thus, the 541 eligible patients from 51 different centers were randomized in different ways, in order to increase the accrual in a pragmatic fashion : 285 were included in a twoby-two factorial design (70 for chemoradiation, 74 for chemotherapy, 72 for both, and 69 for observation only); another 68 patients were randomly assigned to chemoradiation or non chemoradiation and 188 to chemotherapy or no chemotherapy.

Results emerging from the two-by-two factorial analysis (n $=285$ ) did not show longer survival for patients treated with chemotherapy or chemoradiation compared to observation. By contrast, when pooling the results for all the patients included in the different randomization options in a somewhat peculiar way, the authors showed evidence of a survival benefit after adjuvant chemotherapy (median survival :19.5 months for the 238 patients with chemotherapy vs 14.0 months for the 235 patients without, $\mathrm{p}<0.0005)$ On the other hand there was no difference between the median survival of the 175 patients receiving chemoradiation vs the 178 patients without chemoradiation (15.5 vs 16.1 months, $p=0.024)$ (Table I). Major prognostic factors were involvement of the resection margin, tumour grade, tumour size, nodal involvement and administration of chemotherapy.

Should these conclusions affect our view of the role of chemoradiation after surgery for pancreatic cancer? As raised by the accompanying editorial ${ }^{10}$, this study triggered a great deal of criticisms and is subject to all the methodological pitfalls outlined above.

Moreover, because of serious concerns that enough patients might not be available for the $2 \times 2$ randomization in this trial, the investigators pragmatically added two additional ways of randomization : chemotherapy vs no chemotherapy and chemoradiation vs no chemoradiation; they also allowed patients to receive, according to their own or their physician's preference additional "background" chemotherapy or chemoradiation that were not part of the study regimens. Subsequently, the results emerged in three parallel trials in which a high proportion of patients (about 40\%) received therapies that were not defined when the study started. All these modifications undoubtedly led to odd classifications in which "no chemotherapy" patients who had received chemoradiation were compared to "chemotherapy" patients who also had received chemoradiation. The same criticism held true for the "chemoradiation" and "no chemoradiation" groups. In addition, the inclusion criteria were inconclusive for the different groups mentioned Therefore it might be conceivable that, due to the interactions of the different therapies, the study is underpowered and does not allow a proper comparison of the relative regimens.

Consequently, it seems not possible to draw any definite conclusion from the results of the ESPAC-1 trial as regards the efficacy of chemoradiation, which was used in a suboptimal regimen, in the adjuvant setting of pancreatic cancer In addition, recommendation of 5FU/folinic acid regimens, which has displayed extremely low activity in metastatic pancreatic cancer, is also not justified after curative resection as it is unlikely that a treatment that has virtually no activity in advanced disease is active in the adjuvant setting 11,12 .

\section{Neoadjuvant therapy}

The risk of delaying or not receiving postoperative adjuvant treatment prompted many investigators to initiate studies in which chemoradiation using 5FU as a radiosensitizer was given before surgery (Table II). There might be theoretically 
TABLE ॥

Preoperative chemoradiation based on $5 \mathrm{FU}$ regimens in patients with pancreatic cancer

\begin{tabular}{|c|c|c|c|c|}
\hline Author (year) (reference) & No of patients & Treatment & Hospitalization rate & Median survival (months) \\
\hline $\begin{array}{l}\text { Hoffman (1998) } \\
\text { (13) }\end{array}$ & 53 & 50.4 Gy + 5FU + Mitomycin-C & $51 \%$ & 15.7 \\
\hline $\begin{array}{l}\text { Spitz (1997) } \\
\text { (14) }\end{array}$ & 41 & $30-50.4 \mathrm{~Gy}+5 \mathrm{FU} C$ & $32 \%$ & 19.2 \\
\hline $\begin{array}{l}\text { Pisters (1998) } \\
\text { (15) }\end{array}$ & 35 & $30 \mathrm{~Gy}+5 \mathrm{FU}$ & $9 \%$ & 25 \\
\hline $\begin{array}{l}\text { Snady (2000) } \\
\text { (16) }\end{array}$ & 68 & $\begin{array}{l}\text { preop } 40 \text { Gy split + 5FU + } \\
\text { cisplatin + streptozotocin } \\
\text { (20/68 curatively resected) }\end{array}$ & - & 23.6 \\
\hline
\end{tabular}

some tumor-biological advantages of preoperative chemoradiation especially based on short and intensive course of radiotherapy, but so far, the only reports regarding the acceptability and feasability of such therapy concern small nonrandomized series Therefore, most surgeons remain reluctant into using this approach and prefer the post-operative treatment. The potential advantages of preoperative chemoradiation are:

-1 increased cell sensitivity to radiation before surgery due to better oxygenation of the tumor cells

- 2 no delay of postoperative recovery and postponement of the planned treatment

- 3 lower incidence of postoperative anastomotic leaks

- 4 lower rate of positive margin resection (retroperitoneal) due to downstaging

- 5 avoidance of surgery in patients with evidence of rapidly disseminating disease on restaging after chemoradiation $(25 \%)$ and thus better selection of patients who will benefit from pancreatic resection.

Several phase II studies have been published They have shown the feasibility of combining preoperatively radiation (30 to $50 \mathrm{~Gy}$ ) and continuous 5FU infusion (300 $\mathrm{mg} / \mathrm{m}^{2} /$ day $)^{13-15}$. In one study, toxicity was reduced when chemoradiation was delivered over 2 weeks up to a total dose of $30 \mathrm{~Gy}$ (3 Gy/fraction); 20/35 patients underwent successful pancreaticoduodenectomy. Local tumour control and patient survival were equal to the results provided by standard fractionated chemoradiation (50.4 Gy in 5.5 weeks, $1.8 \mathrm{~Gy} /$ fraction); only $10 \%$ of the 20 patients resected had a loco-regional relapse and the median survival for all 20 was 25 months $^{15}$ (Table II). Another non-randomised study reported on the survival advantage of preoperative chemoradiation in patients with regional pancreatic cancer; a first group of 68 patients with proven unresectable pancreatic cancer was given radiation ( $40 \mathrm{~Gy}$ in split course) combined with $5 \mathrm{FU}$, platinum and streptozocin and 20 of them were then curatively resected; another group of 91 patients with resectable pancreatic cancer underwent surgery first followed by chemotherapy \pm radiation in 63 of them. Median survival was 23.6 months in the neoadjuvant group compared to 14 for the patients with initial resectable disease $(p=0.006)$ while the postoperative mortality rates were similar 10 and $5 \%$, respectively $)^{16}$. Although these data were based on a histori- cal control group, they suggest that control of the disease can be improved by downsizing the tumour through neoadjuvant therapy. On the other hand, it should be taken into account that the survival gain emerging from these neoadjuvant studies may simply result from better selection of patients : firstly, preoperatively, by accurate staging and secondly, peroperatively when only the good responders benefited from curative resection.

As toxicity emerging from this approach is moreover not negligeable, it certainly needs further refinement and more data on feasibility before embarking into large randomized trials. Also, the elaboration of neoadjuvant strategies for combined modality treatments calls for the following requirements:

- a proper definition of inclusion criteria

- a proven pancreatic adenocarcinoma (by histology or cytology)

- an accurate definition of resectability of the tumor by a surgical team

- an optimal biliary drainage to avoid morbidity due to cholangitis during chemoradiation.

- Consequently, this strategy should therefore be restricted to clinical trials.

\section{New combined regimens}

Although the modalities of 5FU-based chemoradiation can be further explored (neoadjuvant setting, intensified regimen, continuous infusion for the entire chemoradiation period of 6 weeks), we clearly need more innovative drug regimens in view of the limited activity of 5FU in metastatic pancreatic cancer ${ }^{11,12}$. This is needed in order to achieve, not only a better locoregional tumor control, by maximizing additive or synergistic effects with radiation, but also to treat microscopic extrapancreatic metastatic disease which is responsible of most tumour relapses.

Gemcitabine, a deoxycytidine analogue capable of inhibiting DNA replication and repair, has proved more benefitial than 5FU in advanced pancreatic cancer, with a higher response rate (5.4 vs $0 \%)$, greater clinical benefit ( 23.8 vs $4.8 \%$ ) and longer survival (5.65 vs 4.41 months $)^{17}$. Gemcitabine is also a potent radiosensitizer of human pancreatic cells in vitro (PANC-1) and in vivo models ${ }^{18-20}$. 
TABLE III

Gemcitabine + concomitant radiation: main reports of phases I-II in pancreatic cancer

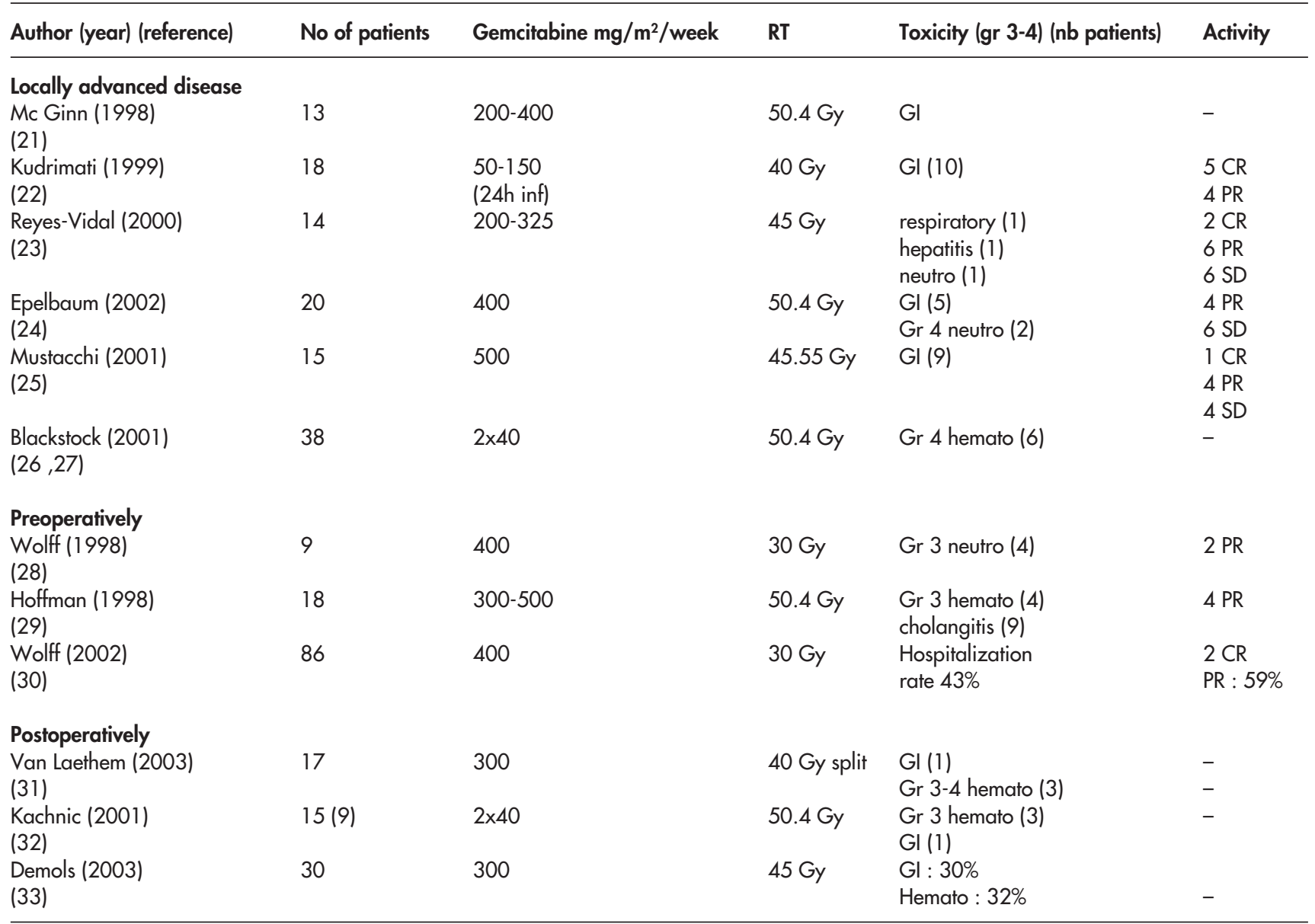

CR: complete response, PR: partial response, SD: stable disease; GI: gastrointestinal; neutro: neutropenia; hemato : hematological; Gr: grade

There is now increasing clinical evidence that gemcitabine administration can be combined with radiation in pancreatic cancer, both in locally advanced disease and in the setting of (neo)adjuvant treatment; the most important phase I and II studies reported so far are shown in Table III. Gemcitabine was mainly administered weekly $\left(300\right.$ to $500 \mathrm{mg} / \mathrm{m}^{2}$ infused over $30 \mathrm{~min}$ IV) or twice weekly $\left(40 \mathrm{mg} / \mathrm{m}^{2} \mathrm{X} 2\right)$ with concomitant radiation (30 to $50.4 \mathrm{~Gy})^{21-33}$. Other approaches included giving gemcitabine at a fixed dose of $1000 \mathrm{mg} / \mathrm{m}^{2}$ while increasing the intensity of the weekly dose of radiation $^{34}$. The overall treatment time was kept constant at 3 weeks ( 15 fractions of radiation) and the total dose was increased from 24 Gy to 42 Gy. The authors recommend 36 Gy in $2.4 \mathrm{~Gy}$ fractions on the basis of tolerance, patterns of failure and survival data.

All these regimens exhibited good feasibility and acceptable, although not negligible toxicity So far, approximately 10 phase I-II studies including a median of 17 patients (range :8-58) have been reported, with a rate of grade 4 toxicity (mainly hematological and gastrointestinal) in less than 20$25 \%$ of the patients, compliance with the treatment of about
$80 \%$ and no major late reactions observed after a follow-up period of 12 to 17 months $26,28,31,32$. The results were quite promising,as complete and partial responses were observed both in locally advanced disease and in the neoadjuvant setting. Feasability of gemcitabine combined with radiation therapy in the neoadjuvant setting was recently reported in a large series of 86 patients $^{30}$. Although manageable, the hospitalization rate related to the toxicity of this regimen was high $(43 \%)$ and underlines the fact that this kind of approach should be unequivocally restricted to appropriate trials. High toxicity rates could be attributed to the rapid-fractionated radiotherapeuthic scheme (3 Gy/fraction) as well as the risk of cholangitis in patients with endobiliary stenting. The activity of such a combination with high pathological response rate is appearing. However a survival benefit has not yet been demonstrated.

Gemcitabine based chemoradiation was also successfully administered in the postoperative adjuvant setting in two reports, showing a good tolerance and no major toxicities 30 , ${ }^{31}$. In addition to our first study, an intensified post operative combined regimen was recently assessed using weekly gem- 
TABLE IV

Ongoing trials in the adjuvant setting of pancreatic cancer

\begin{tabular}{|c|c|c|c|c|}
\hline TRIAL & & Treatment arms & n patients & status \\
\hline $\begin{array}{l}\text { - ESPAC 3: } \\
\text { (phase III) }\end{array}$ & $\mathrm{R}$ & $\begin{array}{l}\text { Observation } \\
\text { GEM } \times 6 \text { cycles } \\
\text { 5FU/FA bolus } \times 6 \text { cycles }\end{array}$ & $\begin{array}{l}330 \\
330 \\
330\end{array}$ & ongoing \\
\hline $\begin{array}{l}\text { - RTOG } 9704 \text { US trial: } \\
\text { (phase III) }\end{array}$ & $\mathrm{R}$ & $\begin{array}{l}\text { GEM ( RT + 5FUc ( GEM } 2 \\
5 F U \text { ( RT + 5FUc ( } 5 \text { FU }\end{array}$ & 59 & closed \\
\hline $\begin{array}{l}\text { - EORTC-FFCD: } \\
\text { (phase II-III) }\end{array}$ & $\mathrm{R}$ & $\begin{array}{l}\text { observation } \\
\text { GEM } \times 2 \text { cycles ( RT + GEM 40/270 }\end{array}$ & $40 / 270$ & $\begin{array}{l}\text { open } \\
\text { April } 03\end{array}$ \\
\hline $\begin{array}{l}\text { - German trial: } \\
\text { (phase III) }\end{array}$ & $\mathrm{R}$ & $\begin{array}{l}\text { Observation } \\
\text { GEM x } 6 \text { cycles }\end{array}$ & $\begin{array}{l}235 \\
235\end{array}$ & ongoing \\
\hline
\end{tabular}

citabine $\left(300 \mathrm{mg} / \mathrm{m}^{2}\right)$ with 45 Gy continuous radiation In $28 / 30$ patients, tolerance and compliance of treatment were quite good and toxicity acceptable $e^{33}$.

A recent study compared the therapeutic index of gemcitabine-based chemoradiation to that of 5FU-based chemoradiation in patients with locally advanced disease ${ }^{35}$. Although it was retrospective, it clearly emphasized that, for comparable patients receiving similar radiation regimen (30 Gy in 10 fractions), severe acute toxicity was significantly higher in the group of patients treated with gemcitabine compared to 5FU ( $23 \%$ vs $2 \%, p<0.0001)$ while recurrence rate and survival were comparable However, a small number of patients with minimal arterial involvement whose disease met the radiographic definition of unresectable disease had margin-negative resections after treatment with gemcitabine-based chemoradiation $^{35}$. Therefore, some selected patients could probably benefit from a such neoadjuvant approach inside clinical trials.

Based on the previously reported data, it seems warranted to use gemcitabine in the adjuvant setting of pancreatic cancer, as this agent may help to control both micrometastatic dissemination and local recurrence when combined with concomitant radiation. However, the above mentioned data suggest that the therapeutic ratio for this type of combination may be narrow It should also be noted that an extremal leve of patients selection has gone into these pilot trials; therefore such combinations are not yet suitable for general use and should be further and more widely evaluated in prospective phase II trials.

Other new agents that will be assessed more deeply in the near future include paclitaxel which has revealed promising activity as a radiosensitizer (response rates of 26-29\% in locally advanced disease) and limited toxicity in preliminary reports $^{36,37}$ and other drugs like cisplatin, oxaliplatin or biological agents targeted to the Epidermal Growth Factor receptor or Her-neu2 receptor in combination or not with radiation $^{38-42}$.

\section{Perspectives in the adjuvant setting}

Previous trials have led to conflicting or underpowered results. The results of large but retrospective and non-randomized single institution experiences, or of randomized, but small trials like those conducted by the GITSG or the EORTC suggest that adjuvant chemoradiation may be beneficial, although the EORTC trial was probably an underpowered positive trial rather than a negative one as mentioned above 5,10 . On the other hand, investigators involved in the large ESPAC-1 trial reported a survival benefit for 5FU-based chemotherapy only, while they claimed that chemoradiation has a confounding negative effect on the benefit of chemotherapy alone"; the criticisms concerning this study have been outlined above and the conclusions drawn from this trial should likely not change our standard practice.

What are the major directions that we have to follow for the creation of new trials in the adjuvant setting? Neoadjuvant strategies seem clearly attractive, mainly to test the tolerance and the activity profile of new coumpounds within the frame of clinical phase II trials. These should also include targeted therapies after identification of,e.g. Epidermal or Vascular-endothelial growth factor receptors Today, it is probably too early to propose a large randomized trial taking into account the possible difficulties to manage such patients preoperatively.

Addressing the postoperative approach, there are still controversies on the definition of the standard treatment for resectable pancreatic cancer. In the US, 5FU-based chemoradiation has been considered standard of care, since the reports of the GITSG trial and the 5FU-based chemoradiation experience This strategy has been incorporated into both arms of the current RTOG phase III trial (Table IV). The question raised by this trial is important: can the addition of gemcitabine- based chemotherapy to chemoradiation increase the median survival from 18 months to 25 months as compa- 
red with a 5FU based chemotherapy; the study is now closed after enrollment of 518 patients and the results of the first data analysis are awaited. In Europe, it is consensually admitted that surgery alone remains the standard of care. As gemcitabine alone is currently the standard treatment of metastatic disease, its impact on survival after curative resection is questionable and two phase III trials are currently in progress. Subsequently to the ESPAC-1 trial, the ESPAC group has now launched the ESPAC-3 trial which aims to randomizing 990 patients in three arms comparing 5FU/folinic acid (Mayo regimen) for 6 months, vs gemcitabine for 6 months vs observation; the purpose of this trial is to strengthen the beneficial role of chemotherapy, since the ESPAC investigators consider that the potential benefit of chemotherapy observed in the ESPAC-1 trial was not clear, because of the apparent negative effect of chemoradiotherapy $A$ randomized German phase III trial aiming to recruit 470 patients is currently exploring the potential beneficial effects of gemcitabine administered alone in an adjuvant setting(TableIV). It is tempting to postulate that a combination of chemotherapy and chemoradiation aimed at controlling both local and distant relapses would be of potential benefit in the adjuvant setting The combination of gemcitabine with radiation is a good candidate to be tested in this setting and will deserve further evaluation in the next future (Table IV). Initial assessment of a good tolerability profile is crucial based on the preliminary results observed in selected centers (Table III) ${ }^{21-33}$.

The results of these trials will be not available for 3 to 5 years. Meanwhile, current and future research is needed to assess the efficacy of new gemcitabine-based drug combinations adding signal transduction-inhibitors or immunotherapeutic agents like this, we will profit for a better understanding of the disease for the sake of our patients.

\section{References}

1. Nitechi SS, Sarr MG, Colby TV et al. Long-term survival after resection for ductal adenocarcinoma of the pancreas: is it really improving? Ann Surg 1995; 221:59-66.

2. Allema JH, Remiders ME, Van Gulik TM et al. Prognostic factors for survival after pancreaticoduodenectomy for patients with carcinoma of the pancreatic head region. Cancer 1995; 75:2069-76.

3. Yeo CJ, Abrams RA, Grochow LB et al. Pancreaticoduodenectomy for pancreatic adenocarcinoma; postoperative adjuvant chemoradiation improves survival. A prospective single institution experience. Am Surg 1997; 226: 621-36.

4. Kalser MH, Ellenberg SS. Pancreatic cancer: adjuvant combined radiation and chemotherapy following curative resection. Arch Surg 1985; 120:899-903.

5. Klinkenbiil JH, Jeekel J, Samouhd T et al. Adjuvant radiotherapy and 5-fluorouracil after curative resection for the cancer of the pancreas and periampullary region: phase III trial of the EORTC Gastrointestinal Tract Cancer Cooperative Group. Am Surg 1994; 230:776-84.

6. Sohn TA, Yeo CJ, Cameron JL, Kouaris L, Koushol S, Abrams RA et al. Resected adenocarcinoma of the pancreas $-616 \mathrm{pa}-$ tients: results, outcomes and prognostic indicators. J Gastrointest Surg 2000; 4:567-79.

7. Mehta VK, Fisher GA, Ford JM, et al. Adjuvant radiotherapy and concomitant 5 -fluorouracil by protracted venous infusion for resected pancreatic cancer. Int J Radiat Oncol Biol Phys 2000; 48:1483-7.

8. Bakkervold KE, Arnesjo B, Dahl O et al. Adjuvant combination chemotherapy (AMF) following radical resection of carcinoma of the pancreas and papilla of Vater: results of a controlled, prospective, randomised, multicenter study. Eur J Cancer 1993; 5:698-703

9. Neoptolemos JP, Dunn JA, Stocken DD et al. Adjuvant chemoradiotherapy and chemotherapy in resectable pancreatic cancer: a randomised controlled trial. Lancet 2001; 358:1576-85.

10. Abrams RA, Lillermoe KD, Piantadosi S. Continuing controversy over adjuvant therapy of pancreatic cancer. Lancet 2001; 358:1565.

11. Van Rijswick RE, Jeziorski K, Wagener DJ et al. Weekly high dose 5-fluorouracil (5FU) and folinic acid (LV) in metastatic pancreatic carcinoma : a phase II study of the EORTC GITCCG. Proc.Am Soc Clin Oncol 2000; 19 :258a (A1002).

12. Rougier Ph, Ducreux $M$, Douillard JY et al. : Efficacy of 5FU+cisplatin (FUP) compared to bolus 5FU in advanced pancreatic carcinoma: a randomized trial from the French Anticancer Centers Digestive group (FNLCCDG) Proc Am Soc Clin Oncol 1999; 18:274a (A1050).

13. Hoffman JP, Lipsitz S, Pisousky T et al. Phase II trial of preoperative radiation therapy and chemotherapy for patients with localized resectable adenocarcinoma of the pancreas. An ECOG study. J Clin Oncol 1998; 16:317-23.

14. Spitz FR, Abruzzese JL, Lee JE et al. Preoperative and postoperative chemoradiation strategies in patients treated with pancreaticoduodenectomy for adenocarcinoma of the pancreas. J Clin Oncol 1997; 15:928-37.

15. Pisters PWT, Abruzzese JL, Janjan NA et al. Rapid fractionation preoperative chemoradiation, pancreaticoduodenectomy and intraoperative radiation therapy for resectable pancreatic adenocarcinoma. J Clin Oncol 1998; 16:3843-50.

16. Snady H, Bruckner H, Cooperman A, Paradiso J, Kiefer L. Survival advantage of combined chemoradiotherapy compared with resection as the initial treatment of patients with regional pancreatic carcinoma. An outcomes trial. Cancer 2000; 89:314-27.

17. Burris HA, Moore MJ, Andersen J et al. Improvements in survival and in clinical benefit with Gemcitabine as first-line therapy for patients with advanced pancreas cancer: a randomized trial. J Clin Oncol 1997; 15:2403-413.

18. Lawrence TS, Change EY, Hohn TM et al. Radiosensitization of pancreatic cancer cells by $2^{\prime}, 2^{\prime}$-difluoro-2'-deoxycytidine. Int J Radiat Oncol Biol Phys 1996; 34:867-72.

19. Milas L,Fujii T, Hunter $\mathrm{N}$ et al : Enhancement of tumor radioresponse in vivo by gemcitabine. Cancer Res 1999; 59:107-14

20. Mason KA, Milas L, Hunter $\mathrm{N}$ et al: Maximizing therapeutic gain with gemcitabine and fractionated radiation Int $J$ Radiat Onc Biol Phys 1999; 44:1125-35.

21. Mc Ginn CJ, Smith DC, Szarka Ce et al. A phase II study of Gemcitabine (GEM) in combination with radiation therapy in patients with localized unresectable pancreatic cancer. Proc Am Soc Clin Oncol 1998; 17:A1014.

22. Kudrimoti $M$, Regine $W$, John $W$ et al. Concurrent infusional gemcitabine and radiation in the treatment of advanced unresectable GI malignancy: a phase I/II study. Proc Am Soc Clin Oncol 1999; 18:A928.

23. Reyes-Vidal J, Rodriguez P, Reyes J et al : Chemoradiation therapy with gemcitabine in advanced pancreatic cancer Proc Am Soc Clin Oncol 2000; 19:A1183. 
24. Epelbaum R, Rosenblatt E, Nasrallah S, et al. Phase II study of gemcitabine combined with radiation therapy in patients with localized, unresectable pancreatic cancer. J Surg Oncol 2002 8:138-43.

25. Mustacchi G, Beorchia A, Milani S, Ceccherini R, Amoroso V Marcuzzi $S$ et al. Gemcitabine and concomitant radiation for locally advanced or relapsed pancreatic cancer. Proc Am Clin Oncol 2001; 20:A2288.

26. Blackstock AW, Bernard SA, Richards F, Eagle KS, Case LD, Poole ME. Phase I trial of twice-weekly gemcitabine and concurrent radiation in patients with advanced pancreatic cancer. J Clin Oncol 1999; 17:2208-12.

27. Blackstock WA, Tempero MA, Niedwiecki D, Hollis DR, Mayer RJ, Teffer JE. Cancer and leukemia Group B 89805: Phase II chemoradiation trial using gemcitabine in patients with locoregional adenocarcinoma. Proc Am Soc Clin Oncol 2001; 20:A627.

28. Wolff RA, Evans DB, Gravel DM et al. Phase I trial of Gemcitabine combined with radiation for the treatment of locally advanced pancreatic adenocarcinoma. Proc Am Soc Clin Oncol 1998; 17:A1091

29. Hoffman JP, Mc Ginn CJ, Szarka CE et al. A phase I study of preoperative Gemcitabine with radiation therapy followed by postoperative Gemcitabine for patients with localized, resectable pancreatic adenocarcinoma. Proc Am soc Clin Oncol 1998; 17:A1090.

30. Wolff RA, Evans DB, Crane $\mathrm{CH}$ et al: Initial results of preoperative gemcitabine-based chemoradiation for resectable pancreatic adenocarcinoma Proc Am soc clin Oncol 2002; 21:130a (A516).

31. Van Laethem JL, Demols A, Gay F, et al. Postoperative adjuvant Gemcitabine and concurrent radiation after curative resection of pancreatic head carcinoma: a phase II study. Int J Radiat Oncol Biol Phys 2003; in press.

32. Kachnic LA, Mornex F, Partensky C, Show J, Neifeld J, Tepper JE et al. Phase II trial of twice-weekly Gemcitabine and concurrent radiation for the treatment of resected pancreatic cancer. Proc Am Soc Clin Oncol 2001; 20: A2334.

33. Demols $A$, Peeters $M$, Polus $M$ et al: Adjuvant gemcitabine and concurrent continuous radiation (45 Gy) for resected pancreatic head carcinoma: a multicenter Belgian phase II study. Proc Am Soc Clin Oncol 2003; in press.

34. McGinn CJ, Zalupski MM, Shureigi I, Roleerstson JM, Eckhouser FE, Smith DC et al. Phase I trial of radiation dose escalation with concurrent weekly full-dose Gemcitabine in patients with advanced pancreatic cancer. J Clin Oncol 2001; 19:4202-8.

35. Crane $\mathrm{CH}$, Abbruzzese JL, Evans DB et al. Is the therapeutic index better with gemcitabine-based chemoradiation than with 5 fluorouracil-based chemoradiation in locally advanced pancreatic cancer? In I J Radiat Oncol Biol Phys 2002; 52:1293-302.

36. Safran $H$, Moore $T$, lanniti $D$ et al: Paclitaxel and concurrent radiation for locally advanced pancreatic cancer Int J Rad Oncol 2001; 49:1275-9.

37. Bertelto O, Di Petrillo T, Safran H, lanitti D, Ackerman P, Cioffi $W$, Quirk $D$ et al. Paclitaxel and concurrent radiation for locally advanced pancreatic cancer. Proc Am Soc Clin Oncol 2001; 20:A1 152

38. Brunner TB, Grabenbauer G, Kastl S et al: a phase I trial of simultaneous gemcitabine/cisplatine and radiotherapy for patients with locally advanced pancreatic adenocarcinoma. Proc Am Soc Clin Oncol 2000; 19:A1 109.

39. Wilkowski R, Heinemann $\mathrm{V}$, Rau $\mathrm{H}$ et al: radiochemotherapy including gemcitabine and 5-Fluorouracil for treatment of locally advanced pancreatic cancer Proc Am Soc Clin Oncol 2000; 19; A1078.

40. Louvet C, André T, G. Lledo, et al: Gemcitabine-oxaliplatin (GEMOX) combination in advanced pancreatic carcinoma (APC): final results of a GERCOR multicenter phase II study. J Clin Oncol 2002; 20(6):1512-8.

41. Abruzzese J, Rosenberg A, Xiong $Q$ et al: Phase II study of antiepidermal growth factor receptor (EGFR) antibody Cetuximab (IMC-C225) in combination with gemcitabine in patients with advanced pancreatic cancer. Proc Am Soc Clin Oncol 2001; 20:A518.

42. Safran H, Ramanathan RK, Schwartz J et al: Herceptin and gemcitabine for metastatic pancreatic cancers that overexpress Her-2/neu. Proc Am Soc Clin Oncol 2001; 20,A517.
Correspondence:

J. L. Van Laethem, MD

Department of Gastroenterology and GI Oncology Hôpital Erasme

Route de Lennik, 808

B - 1070 Brussels

Belgium

E-mail: jvlaethe@ulb.ac.be 